University of Nebraska - Lincoln

DigitalCommons@University of Nebraska - Lincoln

\title{
Lae1 regulates expression of multiple secondary metabolite gene clusters in Fusarium verticillioides
}

\author{
Robert A.E. Butchko \\ USDA-ARS, rbutchko@aggienetwork.com \\ Daren W. Brown \\ USDA-ARS, daren.brown@ars.usda.gov \\ Mark Busman \\ USDA-ARS \\ Bettina Tudzynski \\ Westfälischen Wilhelms-Universität Münster \\ Philipp Wiemann \\ Westfälischen Wilhelms-Universität Münster
}

Follow this and additional works at: https://digitalcommons.unl.edu/usdaarsfacpub

Butchko, Robert A.E.; Brown, Daren W.; Busman, Mark; Tudzynski, Bettina; and Wiemann, Philipp, "Lae1 regulates expression of multiple secondary metabolite gene clusters in Fusarium verticillioides" (2012). Publications from USDA-ARS / UNL Faculty. 1578.

https://digitalcommons.unl.edu/usdaarsfacpub/1578

This Article is brought to you for free and open access by the U.S. Department of Agriculture: Agricultural Research Service, Lincoln, Nebraska at DigitalCommons@University of Nebraska - Lincoln. It has been accepted for inclusion in Publications from USDA-ARS / UNL Faculty by an authorized administrator of DigitalCommons@University of Nebraska - Lincoln. 


\title{
Lae1 regulates expression of multiple secondary metabolite gene clusters in Fusarium verticillioides
}

\author{
Robert A.E. Butchko ${ }^{\mathrm{a}, *}$, Daren W. Brown ${ }^{\mathrm{a}}$, Mark Busman ${ }^{\mathrm{a}}$, Bettina Tudzynski ${ }^{\mathrm{b}}$, Philipp Wiemann ${ }^{\mathrm{b}}$ \\ ${ }^{a}$ National Center for Agricultural Utilization Research, Peoria, IL, United States \\ ${ }^{\mathrm{b}}$ Insitut für Biologie und Biotechnologie der Pflanzen der Westfälischen Wilhelms-Universität Münster, Hindenburgplatz 55, 48143 Münster, Germany
}

\section{A R T I C L E I N F O}

\section{Article history:}

Received 27 October 2011

Accepted 10 June 2012

Available online 17 June 2012

\section{Keywords:}

Fusarium verticillioides

Fumonisin

Secondary metabolism

LaeA

\begin{abstract}
A B S T R A C T
The filamentous fungus Fusarium verticillioides can cause disease of maize and is capable of producing fumonisins, a family of toxic secondary metabolites linked to esophageal cancer and neural tube defects in humans and lung edema in swine and leukoencephalomalacia in equines. The expression of fumonisin biosynthetic genes is influenced by broad-domain transcription factors (global regulators) and Fum21, a pathway-specific transcription factor. LaeA is a global regulator that in Aspergillus nidulans, affects the expression of multiple secondary metabolite gene clusters by modifying heterochromatin structure. Here, we employed gene deletion analysis to assess the effect of loss of a F. verticillioides laeA orthologue, LAE1, on genome-wide gene expression and secondary metabolite production. Loss of Lae1 resulted in reduced expression of gene clusters responsible for synthesis of the secondary metabolites bikaverin, fumonisins, fusaric acid and fusarins as well as two clusters for which the corresponding secondary metabolite is unknown. Analysis of secondary metabolites revealed that, in contrast to a previously described Fusarium fujikuroi lae1 mutant, bikaverin production is reduced. Fumonisin production is unchanged in the F. verticillioides lae 1 mutant. Complementation of the F. verticillioides mutant resulted in increased fumonisin production.
\end{abstract}

(c) 2012 Elsevier Inc. All rights reserved.

\section{Introduction}

The filamentous fungal genus Fusarium includes many species that cause plant disease and produce secondary metabolites (SMs); that is, metabolites that are not essential for growth but that can provide an ecological advantage on some substrates or in some environments. Fusarium SMs also include toxins such as fusaric acid, fusarins and trichothecenes; pigments such as aurofusarin, bikaverin and bostrycoidin; and the plant growth regulators gibberellins. Fusarium verticillioides (Sacc.) Nirenberg (teleomorph Gibberella moniliformis Wineland), commonly associated with maize worldwide, is a causal agent of ear and stalk rot of maize, and can produce a family of toxic SMs, fumonisins, that can contaminate maize kernels destined for food and feed (Marasas, 1996). Fumonisins have been implicated as a cause of human esophageal cancer and neural tube defects (Doko et al., 1995; Franceschi et al., 1990; Rheeder et al., 1992; Suarez et al., 2000; Sydenham et al., 1990; Yoshizawa et al., 1994). They are also responsible for multiple animal diseases including leukoencephalomalacia in equines and lung edema in swine (Colvin et al.,

* Corresponding author. Address: Bacterial Foodborne Pathogens and Mycology Research Unit, USDA, ARS, MWA, NCAUR, 1815 N., University Street, Peoria, IL 61604, United States. Fax: +1 3096816689.

E-mail address: rbutchko@aggienetwork.com (R.A.E. Butchko).
1993; Harrison et al., 1990; Oswiler et al., 1992). Reduction or elimination of fumonisin contamination in food and feed produced from maize is critical to food safety. One strategy to accomplish this goal is to limit fumonisin production in maize by reducing the expression of $F$. verticillioides genes responsible for the synthesis of the mycotoxins.

In fungi, genes directly involved in the synthesis of a given SM or family of structurally similar SMs are typically located adjacent to one another in gene clusters. SM biosynthetic gene clusters generally encode proteins that can be divided into four classes based on their function in SM biosynthesis: polyketide synthases (PKSs), nonribosomal peptide synthatases (NRPSs) and terpene synthases (TSs), that catalyze synthesis of the core structure of SMs; cytochrome P450s and flavin-dependent monooxygenases, dehydrogenases, methyltransferases and acyltransferases that catalyze modification of the core structure; transporters; and transcriptional regulators. In general and regardless of function, genes within SM biosynthetic gene clusters often exhibit similar patterns of expression. This co-regulation within SM biosynthetic gene clusters is influenced by narrow and broad-domain transcription factors (Yu and Keller, 2005). Both types of transcription factors bind to promoter regions of the clustered biosynthetic genes and either activate or inhibit gene expression. Narrow-domain transcription factors are generally encoded by genes located within an SM biosynthetic gene cluster and tend to bind only to promoter 
regions of genes in the cluster. Thus, such transcription factors are considered to be pathway specific in that they directly affect genes in only one SM biosynthetic pathway. In contrast, broad-domain transcription factors are encoded by genes located elsewhere in the genome and can bind to promoter regions in multiple SM clusters as well as to genes involved in other physiological processes. Thus, broad-domain factors are not pathway specific.

The fumonisin biosynthetic gene (FUM) cluster consists of 17 genes and is located on $F$. verticillioides chromosome 1 (Butchko et al., 2003, 2006; Proctor et al., 1999a,b, 2003; Seo et al., 2001; Zaleta-Rivera et al., 2006). Fum21 is a fumonisin pathway-specific regulator; it is a $\mathrm{Zn}(\mathrm{II}) 2 \mathrm{Cys} 6$ zinc binuclear DNA binding protein and activates transcription of other FUM cluster genes (Brown et al., 2007). The nitrogen regulator AreA, the $\mathrm{pH}$ regulator PacC, and the sugar uptake regulator $\mathrm{Zfr} 1$ are broad-domain regulators; they are encoded by genes located outside the FUM cluster but affect FUM gene expression, presumably by binding to FUM gene promoter regions but they also affect other physiological processes (Flaherty et al., 2003; Flaherty and Woloshuk, 2004; Kim and Woloshuk, 2008).

In addition to the FUM cluster, four other biosynthetic gene clusters for four other polyketide derived metabolites (bikaverin, fusaric acid, fusarin, perithecial pigment) have been identified in $F$. verticillioides. The bikaverin biosynthetic gene $(B I K)$ cluster consists of six genes and has been characterized most extensively in Fusarium fujikuroi, a species that is closely related to $F$. verticillioides (Linnemannstons et al., 2002; Brown et al., 2008; Wiemann et al., 2009). The fusaric acid biosynthetic gene (FUB) cluster consists of five genes, the fusarin biosynthetic gene cluster (FUS) consists of nine genes and the perithecial pigment cluster $(P G L)$ consists of seven genes and have recently been described in $F$. verticillioides (Brown et al., 2012b). Each cluster has been characterized by gene expression analysis and in some cases by comparative analysis of genomic DNA sequences. The gene encoding the polyketide synthase required for fusarin production has been characterized in $F$. verticillioides and Fusarium venenatum (Song et al., 2004) and the polyketide synthase required for the perithecial pigment has been described in Fusarium graminearum and Fusarium solani (Gaffor et al., 2005 and Graziani et al., 2004).

In Aspergillus, transcriptional regulation of multiple SM gene clusters can also occur through changes in heterochromatin structure induced by the multi-protein velvet complex, which includes VeA, VelB and LaeA (reviewed in Bayram and Braus, 2011). A key component of this complex, the LaeA protein, is encoded by laeA and was initially identified in a screen of Aspergillus nidulans to identify genes required for synthesis of the mycotoxin sterigmatocystin (Butchko et al., 1999). Subsequent work revealed that LaeA is critical for expression, either positive or negative, of multiple SM gene clusters in Aspergillus as well as in other fungi (Bok and Keller, 2004; Bok et al., 2006a,b; Bouhired et al., 2007; ReyesDominguez et al., 2010; Hoff et al., 2010; Wiemann et al., 2010). In Aspergillus, VeA is required for ascospore production as well as for the production of the SM sterigmatocystin (Bayram and Braus, 2011; reviewed in Calvo, 2008). The VeA homolog in F. verticillioides, FvVe1, has been shown to regulate both SM production and development. Loss of FvVe1 results in alteration of the ratio of microcondia and macroconidia (reviewed in Calvo (2008) and $\mathrm{Li}$ et al. (2006)) as well as the loss of fumonisin and fusarin production (Myung et al., 2009).

Recently, Wiemann et al. (2010) demonstrated that loss of the VeA component of the velvet-like complex in F. fujikuroi affects secondary metabolism, development and virulence on rice plants. Microarray analysis of a Ffvel1 mutant led to the identification of a laeA homolog in F. fujikuroi (Wiemann et al., 2010). Analysis of F. fujikuroi lae1 mutants found that they were similar to the Ffvel1 mutants with respect to reduced gibberellins production and virulence on rice plants, but differ in their effect on bikaverin production (Wiemann et al., 2010).

The objective of this study was to examine the effect of Lae1 on gene expression in $F$. verticillioides. Microarray analysis of a lae1 deletion mutant revealed that Lae1 can positively or negatively affect transcription of multiple SM gene clusters and hundreds of SM-like genes across the $F$. verticillioides genome. In some but not all cases, changes in SM gene expression were accompanied by changes in SM production. To our knowledge, this is the first report of a genome-wide analysis of the effects of the Lae1 on gene expression and SM production in Fusarium.

\section{Materials and methods}

\subsection{Fungal strains and culture conditions}

Fungal strains used are listed in Table 1. Wild-type strain M3125 (NRRL 20960) (Leslie et al., 1992) was the parent strain to the LAE1 deletion strains T21.1 and T22.1. Strains were cultured on V8 juice agar medium for the production of conidia and in liquid GYEP ( $5 \%$ glucose, $0.1 \%$ yeast extract, $0.1 \%$ peptone) for production of mycelia for genomic DNA preparation. Secondary metabolites were assayed from cracked corn medium at 11 days or liquid GYAM (Seo et al., 2001) for 2, 4 and 6 days shaking at $250 \mathrm{rpm}$ at $27{ }^{\circ} \mathrm{C}$ (Desjardins et al., 1996; Seo et al., 2001).

\subsection{Nucleic acid manipulations and fungal transformation}

DNA was isolated using standard protocols (Sambrook et al., 1989). The deletion of the F. verticillioides LAE1 was accomplished using a construct described by Wiemann et al. (2010) to delete the F. fujikuroi LAE1. Southern analysis was used to characterize the deletion strains and the complemented strain. Genomic DNA was digested with EcoRI, separated on agarose gel by electrophoresis, and blotted to nylon membrane. Three different probes were used to characterize the strains. Primer pair rb438 and rb442 was used to amplify a 484 base pair fragment of the LAE1 coding region. Primer pair rb432 and rb435 was used to amplify a 673 base pair fragment corresponding to the region upstream of the LAE1 coding region. Primer pair rb694 and rb697 was used to amplify an 844 base pair fragment corresponding to the region downstream of the LAE1 coding region. DNA fragments were labeled with ${ }^{32} \mathrm{P}$ utilizing Ready-To-Go DNA labeling beads (GE Healthcare, Buckinghamshire, UK). Membranes were hybridized using ULTRAhyb solution (Life Technologies, Grand Island, NY) and washed following manufactures directions. The LAE1 complementation plasmid, Pl-98, was constructed by first amplifying a $2700 \mathrm{bp}$ DNA fragment from genomic DNA of the wild-type strain corresponding to approximately $1 \mathrm{~kb}$ upstream of the predicted Lae1 translational start site and the entire predicted coding sequence using primers rb424 and rb428. The fragment was subcloned into a plasmid containing a gene conferring resistance to geneticin (Marek et al., 1989). The LAE1 fragment was sequenced to determine that no mutations were introduced through PCR amplification. Transformation of LAE1 deletion strain T22.1 was as previously described (Proctor et al., 1999b). Characterization of the add-back strains was accomplished by amplifying PCR products with primers specific to $F$. verticillioides LAE1 coding region.

\subsection{DNA microarrays and RT-PCR}

DNA microarrays (Roche-NimbleGen Systems (Madison, WI)) consisted of approximately 336,000 60-base pair probes, with each sequence in the $F$. verticillioides gene index represented by a set of 6 probes. Each probe is represented twice on each microarray. Total 
Table 1

Fungal strains used in this study.

\begin{tabular}{|c|c|c|c|}
\hline Strain name & Genotype & Antibiotic resistance gene & Reference \\
\hline M3125 & Wild-type & None & Leslie et al. (1992) \\
\hline $\mathrm{T} 21.1$ & LAE1 deletion & Hygromycin & This study \\
\hline $\mathrm{T} 22.1$ & $L A E 1$ deletion & Hygromycin & This study \\
\hline GMTcp-263 & LAE1 deletion, $L A E 1$ add-back & Hygromycin, geneticin & This study \\
\hline GMTcp-264 & LAE1 deletion, LAE1 add-back & Hygromycin, geneticin & This study \\
\hline
\end{tabular}

RNA was isolated from 2, 4 and 6 day liquid GYAM cultures using the RNeasy Mini kit (Qiagen Inc., Valencia, CA) and DNA was removed using the RNase-Free DNase kit (Qiagen Inc., Valencia, CA) Nucleotide labeling, hybridization and data acquisition were done by NimbleGen Systems. Raw intensity values were normalized to internal controls on each chip and between chips using NimbleScan Bioanalyzer software, utilizing Robust Multichip Analysis (Irizarry et al., 2003). Normalized data was analyzed using Acuity 4.0 from Molecular Devices Corporation (Sunnyvale, CA) and P values were generated by a Student's $T$-test using the same software. Differences in gene expression are considered significant at a $P$ value less than 0.05 . Samples of RNA were extracted from three biological replicates of $F$. verticillioides strains cultured in liquid GYAM. End-point reverse transcription PCR (RT-PCR, 30 cycles) was used to assay gene expression. Stratagene (Santa Clara, CA) Easy-A One-tube RT-PCR kits were used following manufactures protocol. Primers are listed in Table 2.

\subsection{Metabolite analysis}

Fumonisin B1, B2 and B3 (Plattner et al., 1996), fusarin C (Maragos et al., 2008) and bikaverin (Vesonder et al., 1989) standards were prepared as previously described. To assess toxin production, strains were grown in triplicate for 6 days in liquid GYAM medium or on cracked corn medium for 11 days. Cracked corn cultures were extracted with acetonitrile:water $(1: 1)$ as previously described (Proctor et al., 1999b). Care was taken to utilize conditions that would minimize decomposition of fusarin $C$ in all samples (Maragos et al., 2008; Kleigrewe et al., 2011). The fumonisin, fusarin C and bikaverin content of liquid culture media or cracked corn

Table 2

Primers used in this study.

\begin{tabular}{lll}
\hline Primer name & Gene & Sequence \\
\hline rb291 & TEF1 & ATGGGTAAGGAGGACAAGAC \\
rb292 & TEF1 & GGAAGTACCAGTGATCATGTT \\
rb371 & FUM21 & GCCAAACGTGCCCTTGGTTCTTA \\
rb374 & FUM21 & TGCATCTTGCCCTACTCACAGC \\
rb375 & FUM1 & TTTGTCGAAATTGGCCCTCACAGC \\
rb378 & FUM1 & ACAACGTCATTGAGGACCTGGTGA \\
rb405 & FvVE1 & CTTTGATCTGGAACCTGCCG \\
rb456 & FvVE1 & GCTGGTCTATCTTGAGAGCC \\
rb414 & BIK1 & AGTGTTGGCAGACATCACC \\
rb416 & BIK1 & CGCTTTGGCAATTTACCGAG \\
rb436 & LAE1 & GGATTCTGGCAACATGGCCG \\
rb440 & LAE1 & AAGTCCGTTTAATACTGCC \\
rb424 & LAE1 & AACCCAAGGACAATTGTGCTG \\
rb428 & LAE1 & CACCAGGCCGATTTGGATTT \\
rb473 & LAE1 & CTTCATTGGGACATTTGGATTT \\
rb445 & HYGB & CCAAGGACTATGACACTGGC \\
rb464 & HYGB & TAGCAGCACGCCATAGTGA \\
rb432 & lae1 & CAAATATCGGCTTAAACGCC \\
rb435 & lae1 & CGGCCATGTTGCCAGAATCC \\
rb438 & LAE1 & GCCAAAACAATATGACATTG \\
rb442 & LAE1 & CCAGACTATGAGACAGCCCG \\
rb694 & lae1 & CAACGCAAGGAACACGAA \\
rb697 & lae1 & TGTCCACCACCTTCTGCTT \\
\hline
\end{tabular}

extracts was determined by liquid chromatography-mass spectroscopy (LC-MS) or liquid chromatography-tandem mass spectroscopy (LC-MS/MS). Analyses were conducted with a LCMS/MS system composed of a ThermoSpectraPhysics high performance liquid chromatography system, consisting of a P4000 pump and AS3000 autosampler, coupled to a ThermoFinnigan DECA ion trap MS operated in positive electrospray ionization mode. Filtered liquid culture medium or cracked corn extract samples were injected into a linear gradient flow on a Metachem (Torrance, CA USA) Inertsil $\mathrm{C} 18(150 \times 3 \mathrm{~mm})$ column. For all analyses a $300 \mu \mathrm{L} / \mathrm{min}$ water/methanol $(\mathrm{MeOH})$ gradient program $(0 \mathrm{~min}$ : 35\% $\mathrm{MeOH}, 5 \mathrm{~min}: 35 \% \mathrm{MeOH}, 15 \mathrm{~min}: 95 \% \mathrm{MeOH}, 20 \mathrm{~min}: 95 \%$ $\mathrm{MeOH}, 25$ min: $35 \% \mathrm{MeOH}$ ) with $1 \%$ acetic acid was used. LC-MS and LC-MS/MS quantitations were made by monitoring a 2 Thomson (Cooks and Rockwood, 1991) window around the $m / z$ of the ion of interest. LC-MS determination of fumonisins was achieved by monitoring $m / z 722$ (FB1) and 706 (FB2 and FB3) protonated $([\mathrm{M}+\mathrm{H}]+)$ ions as previously described (Plattner et al., 1996). Quantitation was done by comparison of the FB1, 2 and 3 chromatographic peak areas to those for fumonisin concentration standards. LC-MS/MS determination of fusarin $\mathrm{C}$ was done by monitoring several distinctive fragments $(\mathrm{m} / \mathrm{z} 364,382,394)$ of the $[\mathrm{M}+\mathrm{H}]+$ ion $(\mathrm{m} / \mathrm{z} 432)$ in manner similar to that previously described (Maragos et al., 2008; Kleigrewe et al., 2011). Quantitation was based on comparison of the chromatographic peak area for the $\mathrm{m} / \mathrm{z} 364$ ion to those for fusarin C concentration standards. LC-MS/ MS determination of bikaverin was done by monitoring several distinctive fragments $(\mathrm{m} / \mathrm{z} 337,355,365)$ of the $[\mathrm{M}+\mathrm{H}]+$ ion $(\mathrm{m} / \mathrm{z}$ 383) (Busman et al., submitted for publication). Quantitation was based on comparison of the chromatographic peak area for the $\mathrm{m} / \mathrm{z} 355$ ion to those for bikaverin concentration standards. Quantitative assessments of the LC-MS and LC-MS/MS experiments were made using the Xcalibur software supplied by the manufacturer of the MS instrument. Microsoft Excel (Redmond, WA) was used for statistical analysis and $\mathrm{P}$ values were generated using a Student's T-test.

\section{Results}

\subsection{Identification and deletion of F. verticillioides LAE1}

In the Fusarium Comparative Database (Brown et al., 2005), LAE1 is designated as FVEG_00539 and is located on chromosome 1. The predicted amino acid sequence of the $F$. verticillioides LAE1-encoded protein (Lae1) is $70 \%$ identical to F. fujikuroi Lae1 and $32 \%$ identical to A. nidulans LaeA. Two lae1 deletion mutants were generated by transformation of wild-type $F$. verticillioides strain M-3125 with a vector engineered for deletion of the $F$. fujikuroi LAE1 homolog (Wiemann et al., 2010) and are designated T21.2 and T22.1 (Fig. 1).

\subsection{Genome-wide gene expression in lae1 mutant}

We employed microarray analysis to compare genome-wide gene expression in lae1 mutant strain GMTcp-258 and wild-type 
(A)
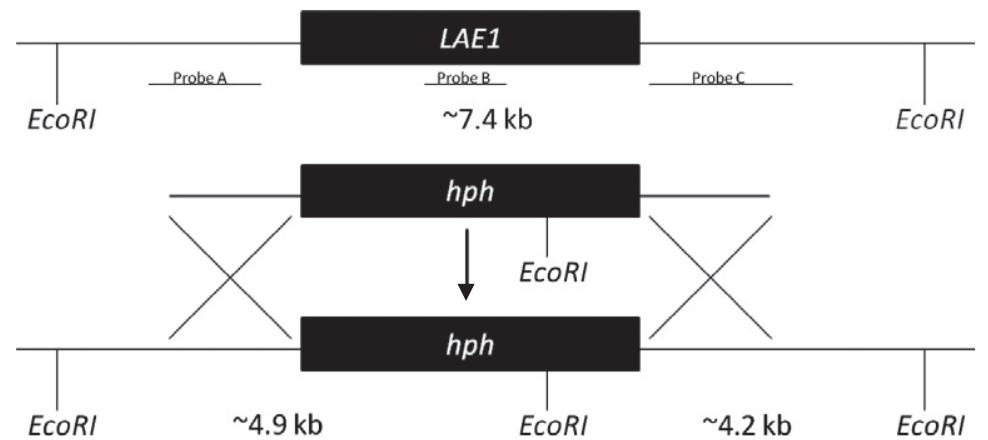

(B)

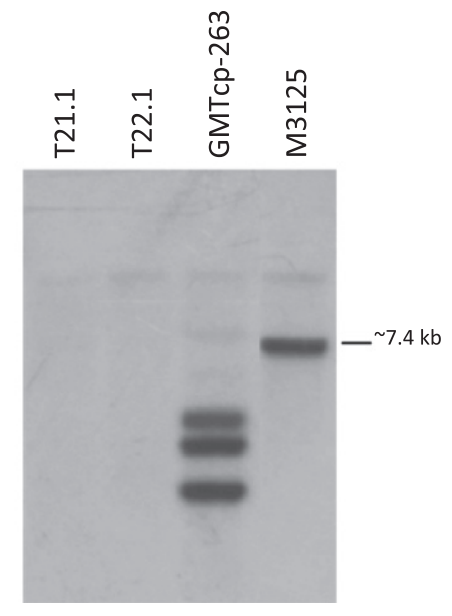

Probe $B$

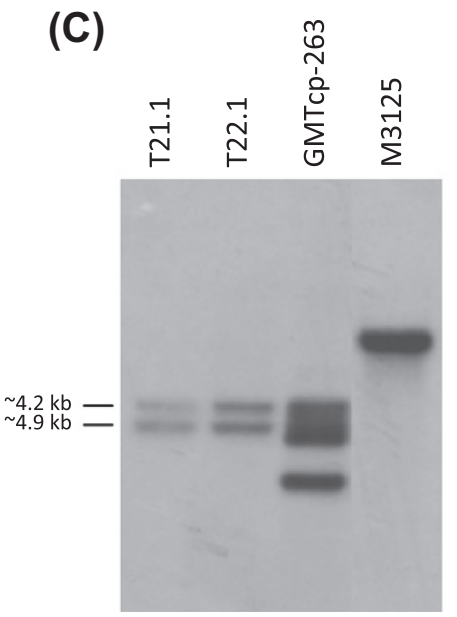

Probes $\mathrm{A}$ and $\mathrm{C}$

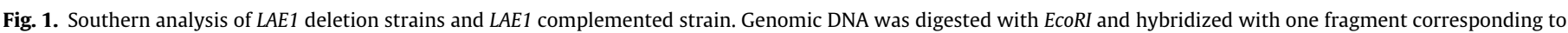

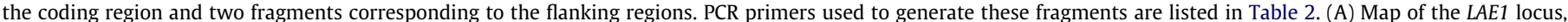

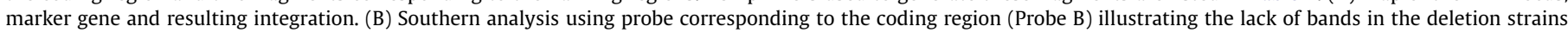

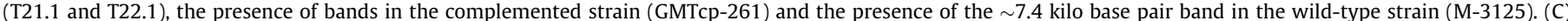

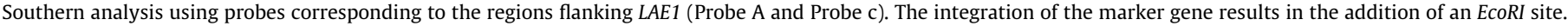
Corresponding bands ( $\sim 4.2$ and $\sim 4.9$ kilo base pairs) are present in the LAE1 deletion strains as expected.

F. verticillioides after 6 days of growth in a liquid medium, GYAM, that is favorable for production of fumonisins and some other secondary metabolites. Microarray data have been deposited in NCBI's Gene Expression Omnibus (Edgar et al., 2002) and are accessible through GEO Series accession number GSE32608 (http:// www.ncbi.nlm.nih.gov/geo/query/acc.cgi?acc=GSE32608). A total of 7910 genes of the 14,196 genes represented on the microarray exhibited expression that was significantly $(P<0.05)$ different in the lae1 deletion mutant compared to its wild-type progenitor strain. Of these genes, 4800 exhibited reduced expression and 3110 exhibited increased expression in the lae1 mutant. Blastp analysis with the National Center for Biotechnology Information (NCBI) database revealed that many of these genes in the subsets described below have homology to genes that are likely involved in SM biosynthesis, including genes encoding monooxygenases, dehydrogenases, reductases, and Zn2(II)Cys6 zinc binuclear DNA binding transcription factors.

Among the 4800 genes that exhibited reduced expression, 201 exhibited at least fourfold reduction in expression (Supplemental Table 1). These 201 genes included 10 sets of two adjacent genes and 10 sets of three or more adjacent genes. Three of the latter sets corresponded to the bikaverin (BIK), fusaric acid (FUB), and fusarin (FUS) biosynthetic gene clusters (Fig. 2). Another set includes four genes without significant homology to genes in the NCBI database, and a fifth set located $15 \mathrm{~kb}$ from the FUB gene cluster includes seven genes with significant homology to genes likely to be involved in SM biosynthesis (Fig. 2D).

Within the group of 3110 genes that exhibited increased expression in the lae 1 mutant compared to wild-type $F$. verticillioides, 74 exhibited at least fourfold greater expression and included seven sets of two or more adjacent genes (Supplemental Table 2). One group included a PKS gene (FVEG_00079) for which the corresponding polyketide-derived metabolite is not known. Forty-two of the 74 genes share significant similarity to genes of unknown function (e.g., hypothetical proteins) by BLASTP while 18 share similarity to genes that are or are predicted to be involved in secondary metabolism (e.g. dehydrogenases, methyltransferases, transporters, and monooxygenases). Interestingly, three of the four genes with homology to methyltransferases also shared homology to laeA-like genes in Aspergillus. An alignment of the deduced amino acid sequence of LAE1 and the four other $F$. verticillioides methyltransferases genes revealed that they all include the putative HemK domain that is characteristic of the S-adenosyl-L-methionine-dependent methyltransferase superfamily (Fig. 3).

\subsection{Expression of PKS genes and their flanking genes}

The $F$. verticillioides genome contains 16 polyketide synthase genes, including the PKS genes required for synthesis of bikaverin (BIK1), fumonisin (FUM1), fusaric acid (FUB1), and fusarins (FUS1) 
(A)

FUM gene cluster

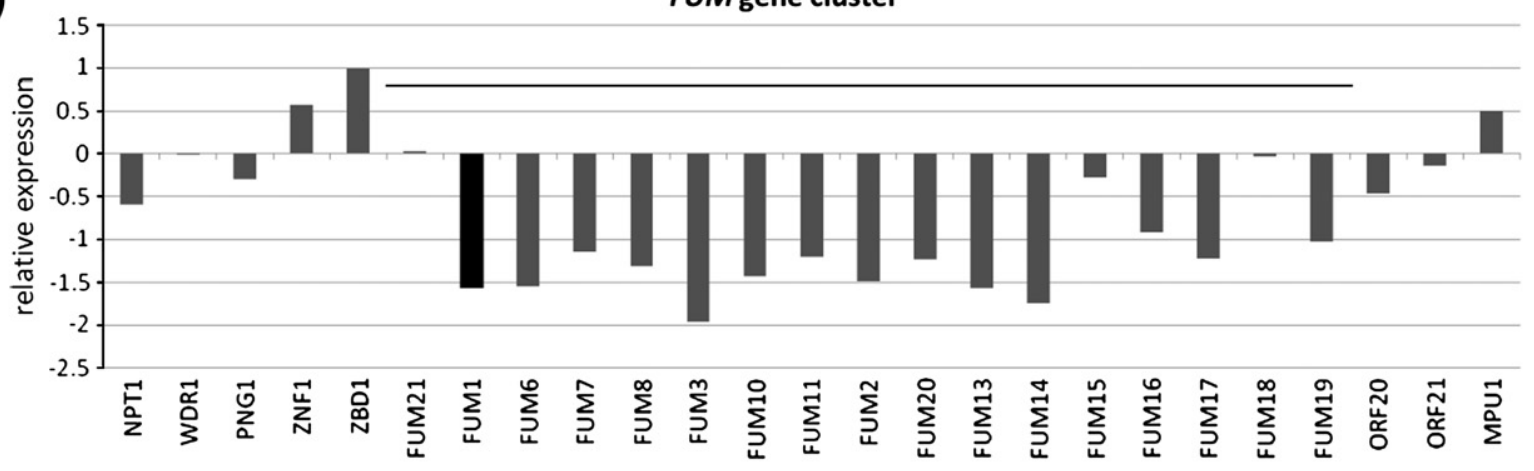

(B)

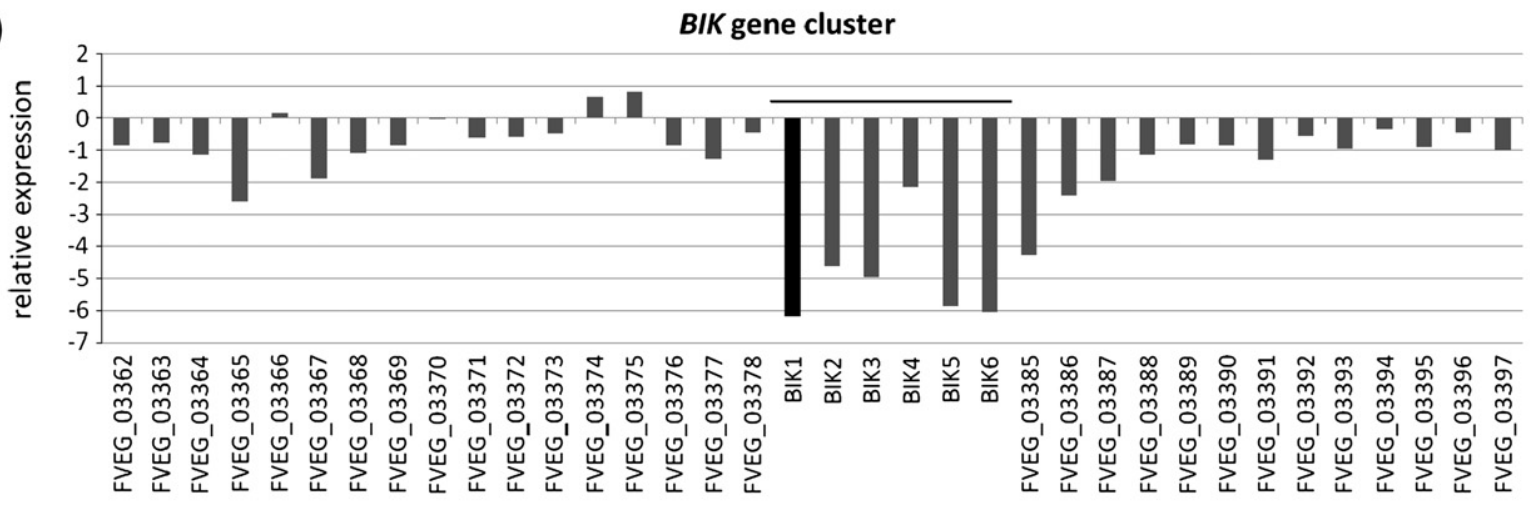

(C)

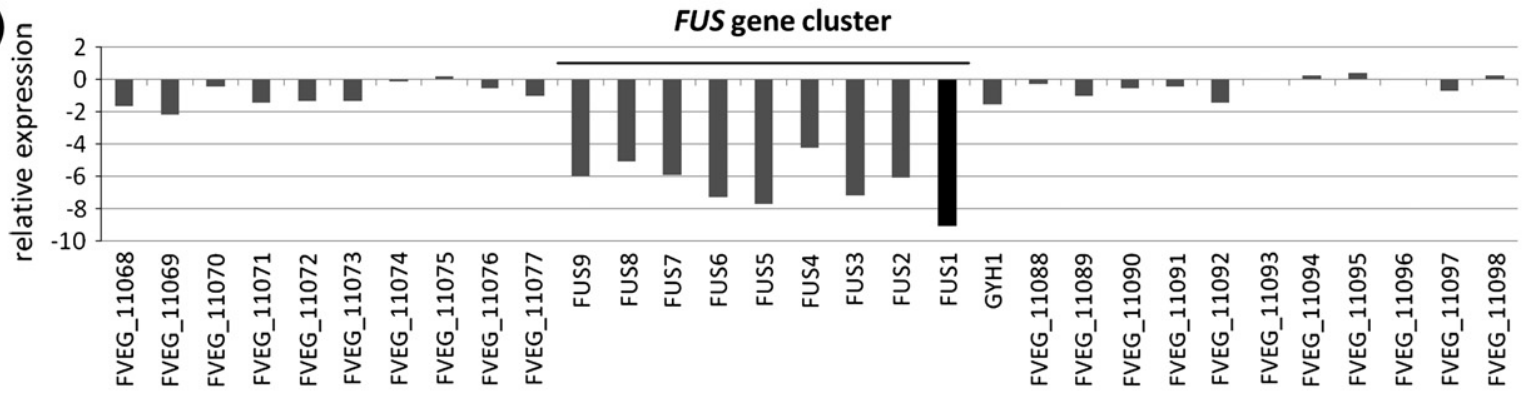

(D)

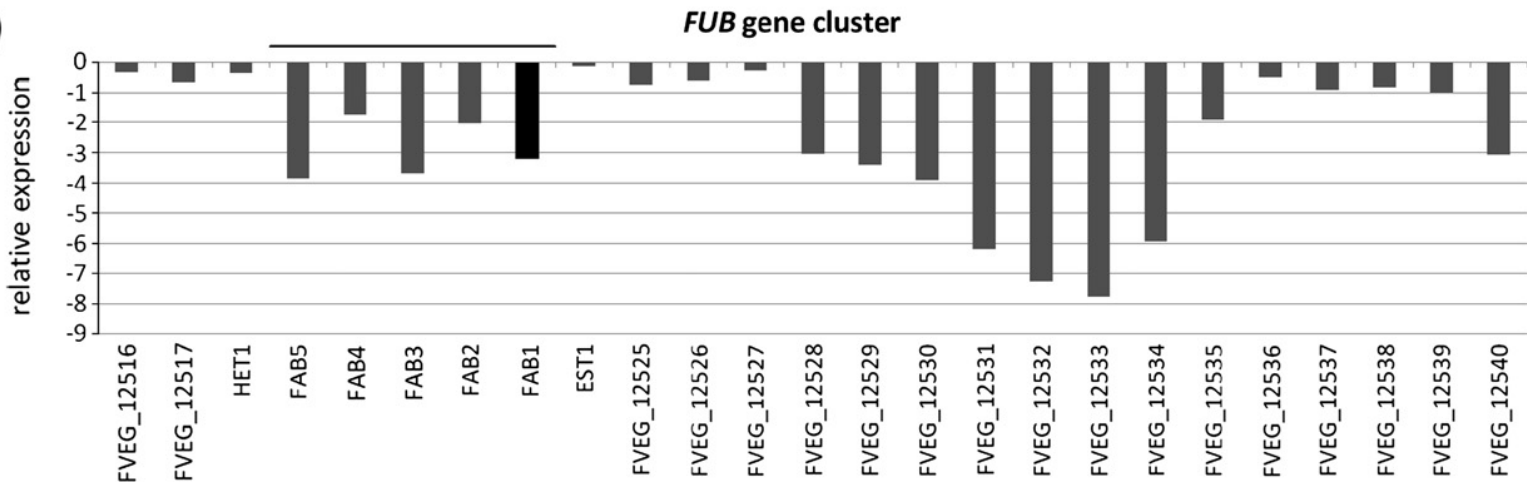

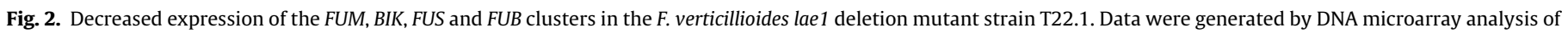

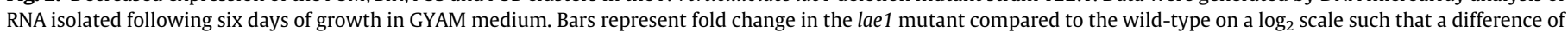

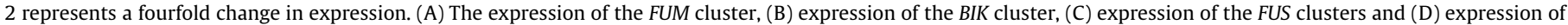
the FUB cluster. Horizontal lines represent the extent of the gene cluster. In each case, the PKS gene is shaded black.

(Ma et al., 2010; Brown et al., 2012b). In order to identify novel SM gene clusters responsible for synthesis of polyketides, we analyzed expression of the PKS gene and surrounding genomic regions in the lae1 mutant compared to the wild-type progenitor strain M-3125. The genomic regions examined included all predicted genes within
40 kilobase pairs $(\mathrm{kb})$ of either side of the PKS gene. In many cases, the predicted function of flanking genes are unknown; in the Broad Institute's Fusarium Comparative Database these genes with unknown functions are annotated as "predicted proteins" or "conserved hypothetical proteins", and in blast analyses against the 
LAE1

FVEG_10732

FVEG_12679

FVEG_13182

LAE1
FVEG_10732
FVEG_12679
FVEG_13182

LAE1

FVEG_10732

FVEG_12679

FVEG_13182

LAE1

FVEG_10732

FVEG_12679

FVEG_13182

LAE1

FVEG_10732

FVEG_12679

FVEG_13182

LAE1

FVEG_10732

FVEG_12679

FVEG_13182

LAE1
FVEG_10732
FVEG_12679
FVEG_13182
$-------------------------------M V V M P P Q----------------\mathrm{N}$ ----------------------MAEPT-INVEPDLVPDEQSEEEHDSSIGSSLA-MDKTDAVSQTNPRFPTEILPESTPPGMSSQGGQDLVAAQPLEENEEDDDDSALGQDFA-- - - - - - - - - - - - - - - MAQEA-NEHESPLQADDAASLSHDSALAASLESG

SVNESEGRYLQDGFWQHGRFYGSWK-PGKYLFPIDSEELNRLDIFHKVFLLARDNKPFLA STSESLRSSILDYRKENGRTYHRYK-DGKYNLPNDDVEKERLDLQHNLF ILMLDNKLGLA SSTASLTSSILEYRKFQGRTFNSDKYEIEYFAPNDERQKESIDISHHYLMLLLDGKLSLA SYLTSLNSSVLNYKYENGRRYHAFR-EGVYLVPNDDEEQDRMDLGHHIYRLLLGGDLFLA

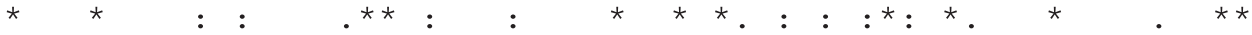

PIR---RTSPRIMDIGTGTGIWAINVAEECLSDAQIMAVDLNQIQPALIPPGFMPKQYDI PPNNPNSKAKRVLDVGTGTGIWAIDYADEH-PEAKVIGVDLSPIQPDFVPPNVEFFIDDI PSP---EDFEKVLDIGTGTGIWAIDFADQY - PNAEVVGTDLSPIQPDWVPPNVRFELEDA PIG---DKVKRVLDLGTGTGIWAMDFADEY-PQAEVLGTDLSPIQPPWTPPNCVFEVDDF

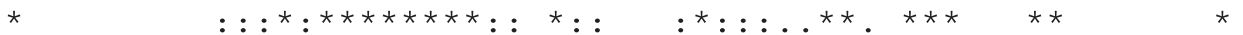

EEPSWGPLLADCDLIHMRMLLGSIQTDLWPQVYHNAFEHLAPGIGFLEHIEVDWIPRCDD E-EPWNFS-EPFDYVHSRMMTFSIK--SWPNLASSIYDNLVPG-GYVELLEIDLFAHSDD T-SNWTWSNDTFDFVHMRYLIGAIA--DWGALFKDAFRCCKPG-GFVESVEVNPTFF SDD E-SDWLFR-QPFDF IHARELEGCIS--NNAQFFTRALASLAPG-GYLEMQAVHSEFKSDD

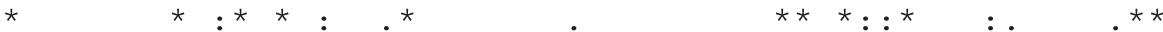

DERPANSAFVKWAELFLDGMDRFNRRVRVTPQEHRQMLEATGFTDIRQEVIKAYVCPWSA KTLTEDHHLSKLIHLLDEASTKIGRPFQDNKKN-KDILRDAGFVDIVETVFKWPTNRWPL GTASDVTAVQTWNKLFREASKAFGRSFCEIEGD-AELLAAAGFVDLQVTDFKVPVGGWAK NTKEKAKNALLWMKNMVEGSSKFGKPLNVAPGW-KKEMEDAGFVDVEQKILKVPIGSWPR

DRNEREIARWFNIGLSH--SLEAMSLKPLIEKLGFEAEEVRELCERAKRETCVLRYHTYC DKRYKEIGEWNNLNMDSFKGLEALSMAALTRVLGWTQEEVTVFLAKVRRDLNDRTIHAYW DSKLRQVGEFLRATMEN--DLEGYTLLAWQQILGWPKDEYQLFLMDMRKALRDKKVHSYI DPKLKEIGKFQSVQEAQ--VITSYTPGIFSRVLGWSDEEIQVFMAKVKKDLSNPSIHLYL * . : : : . : : . $* *: \quad * \quad: \quad:$ : * *

$\mathrm{NM} *--------$

PVYSTYGRRPLD

RVRFLNAHKP--

PVYFIWGRKPE-

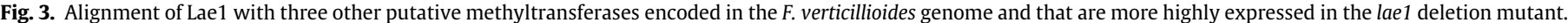

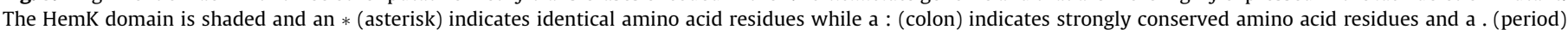

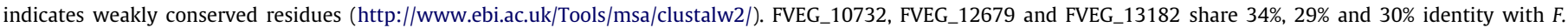
verticillioides LAE1 respectively and exhibit 36\%, 28\% and 32\% identity to A. nidulans LaeA respectively.

NCBI database, done as part of the current study, these genes do not exhibit significant identity to genes with known functions.

We found that 14 PKS genes exhibited significantly decreased expression while two exhibited significantly increased expression in the lae 1 mutant relative to wild-type (Supplemental Table 3). Four of the PKS genes with significantly decreased expression are BIK1, FUB1, FUM1 and FUS1. Analysis of the 40$\mathrm{kb}$ regions on either side of these four genes revealed that numerous genes in the flanking regions also exhibited reduced expression, including genes corresponding to the BIK, FUB, FUM and FUS clusters (Fig. 2). Co-expression of genes in the $B I K$, FUB, FUM and FUS clusters observed for the wild-type progenitor strain in the current study is consistent with previously reported co-expression of the genes (Proctor et al., 1999b; Brown et al., 2012b). In the current experiment, the expression of all FUM genes, except the regulatory gene FUM21, was approximately threefold lower in the lae1 mutant (Fig. 2A). The expression of the six BIK genes, the nine FUS genes and the five FUB genes were 16 to $>500$ fold less in the lae1 mutant (Fig. 2B-D). Although expression of the other 10 PKSs were between 2 and 6-fold less in the lae1 mutant, genes flanking these other PKS genes did not exhibit patterns of changed expression indicative of gene clusters as is the case for the BIK, FUB, FUM and FUS clusters.

The two PKS genes that exhibited increased expression, a 2-fold increase, in the lae 1 mutant compared to wild-type are designated FVEG_00079 and FVEG_10535 in the F. verticillioides genome database (Fig. 4). Three genes flanking FVEG_00079 also exhibited a >2 to 6 -fold increase in expression in the lae 1 mutant. These flanking genes, FVEG_00076, FVEG_00077 and FVEG_00078, are predicted 
(A)

FVEG_00079

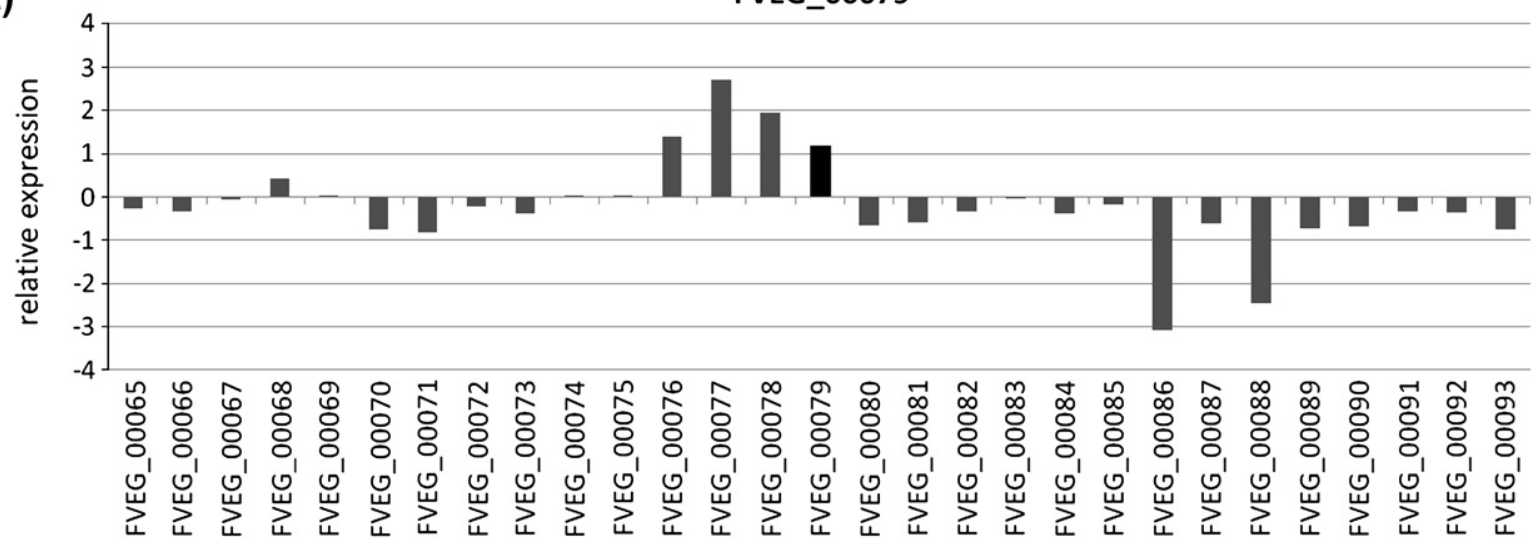

(B)

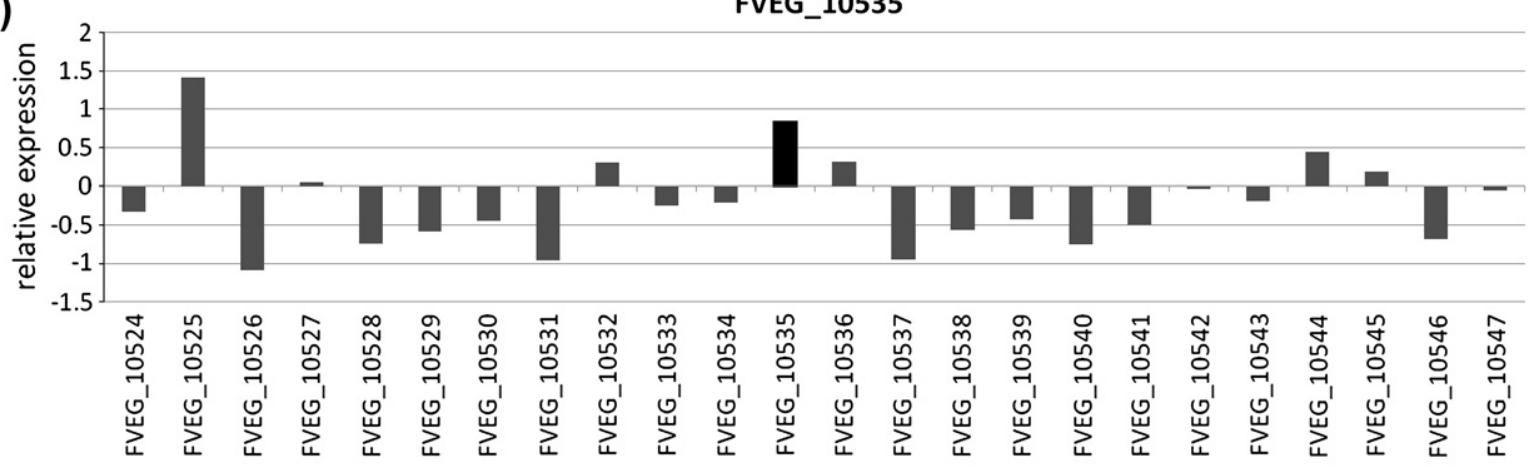

(C)

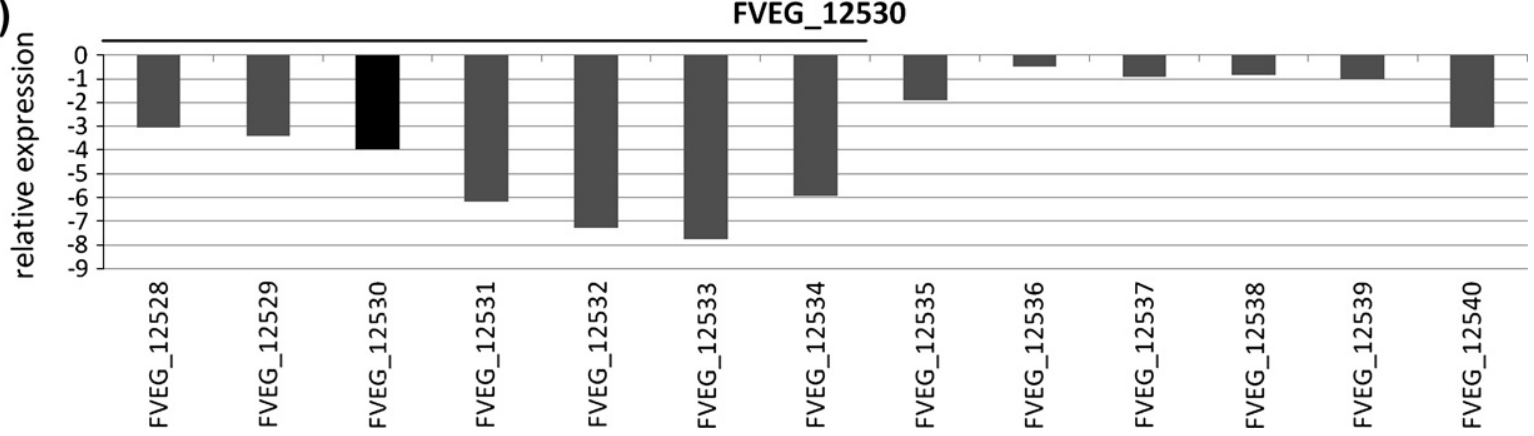

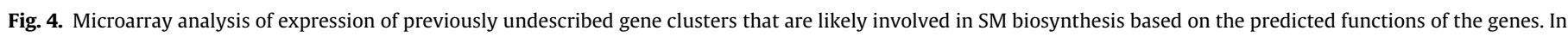
each case, the PKS or NRPS gene is shaded black.

to encode a protein of unknown function, an acetyltransferase, and an integral membrane protein respectively. The co-expression of these flanking genes combined with the close proximity of a PKS gene and the acetyltransferase are consistent with these genes constituting a polyketide-derived SM biosynthetic gene cluster. In contrast, the lack of co-expression of the genes flanking the PKS gene FVEG_10535 (Fig. 4B) provides no support for this gene being part of an SM biosynthetic gene cluster.

\subsection{Identification of putative gene cluster with an NRPS-like gene}

The genome-wide expression analysis also revealed that seven genes located $15 \mathrm{~kb}$ upstream of FUB1 exhibited more than 4-fold decreased expression in the lae 1 mutant compared to wild-type F. verticillioides (Fig. 4C). In the Fusarium Comparative Database, these genes are designated FVEG_12528 through FVEG_12534 and exhibit sequence homology to genes involved in secondary metabolism. FVEG_12532 and FVEG_12534 are predicted to encode Zn2(II)Cys6 binuclear DNA binding domain proteins; FVEG_12528 and FVEG_12531 are predicted to encode dehydrogenases; FVEG_12529 is predicted to encode a lyase; FVEG_12530 is predicted to encode a NRPS-like protein; and FVEG_12533 is predicted to encode a major facilitator (MFS) transporter. The coexpression of these SM-like genes suggests that they may constitute a SM biosynthetic gene cluster.

\subsection{Complementation of LAE1 deletion strain}

The F. verticillioides lae 1 deletion mutants were genetically complemented with a wild-type copy of $F$. verticillioides LAE1. Two independent $L A E 1$ add-back strains were identified by PCR with primers specific to $L A E 1$ coding sequence and are designated GMTcp-263 and GMTcp-264. Expression of LAE1, FUM1, FUM21, $B I K 1, V e 1$ and TEF1 (transcription elongation factor) in the lae1 mutant were compared to the add-back transformant GMTcp263 by end point reverse transcription PCR (RT-PCR) over time. 
As expected, expression of the bikaverin and fumonisin PKSs (BIK1 and FUM1 respectively) were restored in the LAE1 add-back strain (Fig. 5). Expression of Ve1 was not altered in the lae 1 mutant strains and LAE1 expression was restored in the add-back strain. No morphological differences with regard to growth rate or conidiophore production on solid media between the lae 1 mutants or the complemented mutants and the wilt-type strain were observed.

\subsection{Secondary metabolite analysis}

Analysis of production of SMs in the two lae1 mutant strains revealed no statistically significant reduction in fumonisin production while bikaverin and fusarin production were both reduced compared to wild-type (Fig. 6A). In liquid culture, no fusarins were detectable. On cracked corn, bikaverin production was reduced by $70 \%(P<0.01)$. Complementation of the lae1 mutant resulted in a $50 \%$ increase $(P<0.05)$ in fumonisin production compared to wild-type $F$. verticillioides. In contrast, bikaverin production was restored only to approximately 40\% $(P<0.05)$ of wild-type levels while fusarin $C$ was restored to approximately $10 \%(P<0.05)$ in the lae 1 mutant complemented strain (Fig. 6B and C). Fig. 7 shows representative chromatograms from LC-MS or LC-MS/MS measurements of fumonisin (A), bikaverin (B) and fusarin C (C) production.

\section{Discussion}

In Aspergillus species, the velvet complex (composed of the proteins VeA, VelB and LaeA) is involved with the regulation of secondary metabolism and development by affecting gene expression at the chromatin level (Bayram et al., 2008; Bok and Keller, 2004; Bok et al., 2006a,b; Reyes-Dominguez et al., 2010). A recent review catalogs the prevalence of the velvet complex across the fungal kingdom and is reported to be present in Basidiomycetes, Hemiascomycetes, Zygomycete and Chytridiomycetes (Bayram and Braus, 2011). One goal of studies on the velvet complex in diverse fungi is to discover new strategies to control SM synthesis in order to limit mycotoxin contamination of crops and to discover new fungal natural products with novel biological activities. It is clear that although the general function of the velvet complex is conserved, its relative impact on the synthesis of different SMs is species specific within a genus. Here, we describe the ef-
(A)

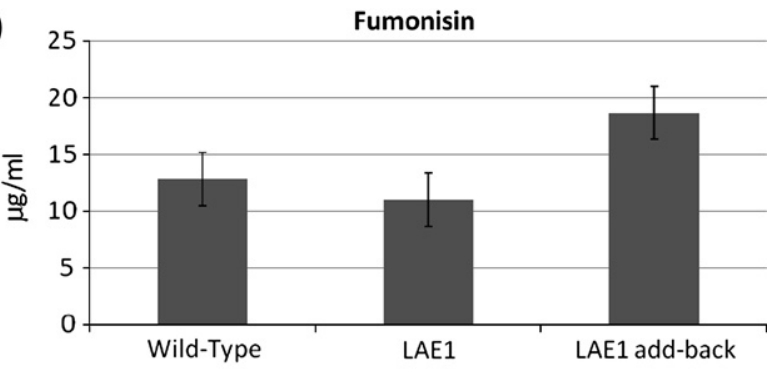

(B)

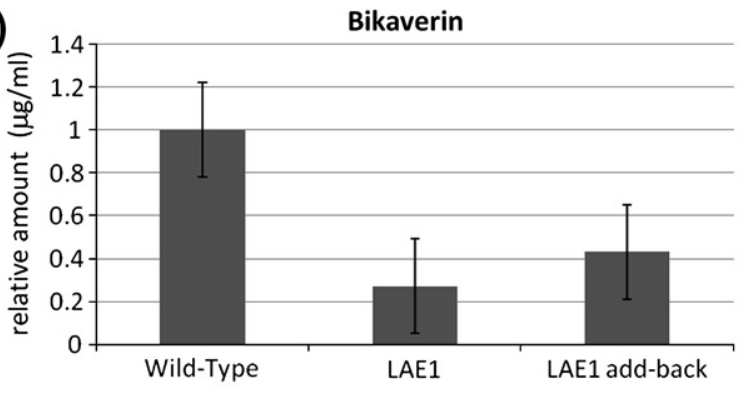

(C)

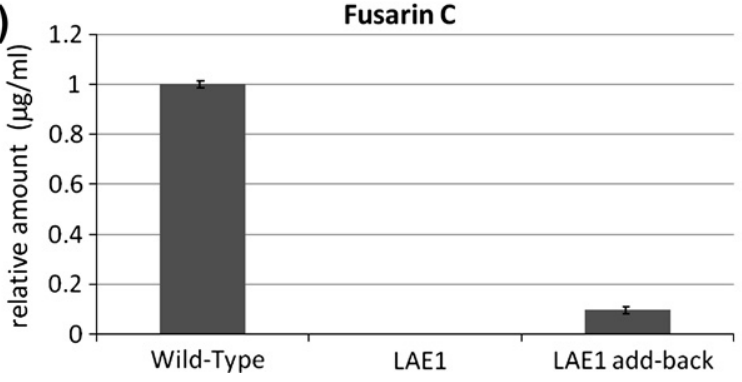

Fig. 6. Secondary metabolite analysis. Fumonisin production (A), bikaverin production (B), and fusarin C production (C) in wild-type (M-3125) LAE1 deletion strain T22.1 (LAE1) and LAE1 complementation strain GMTcp-263 (LAE1 Addback).

fects of loss of Lae1 in F. verticillioides by analysis of deletion mutants. Loss of Lae1 resulted in the loss of expression of the FUM, BIK, FUS and FUB gene clusters and one previously undescribed gene cluster containing an NRPS-like gene (FVEG_12530) and the increase in expression of another previously undescribed
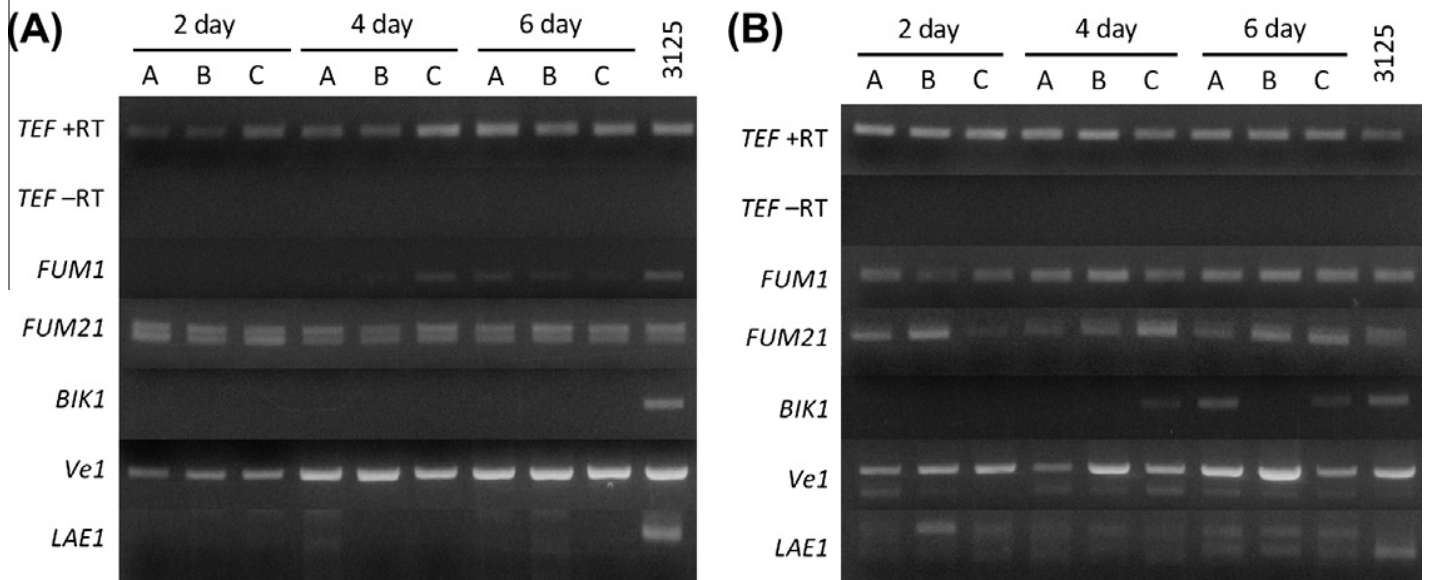

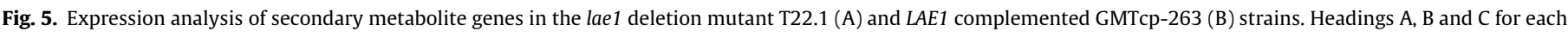

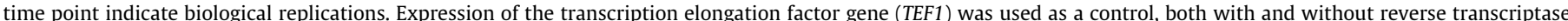

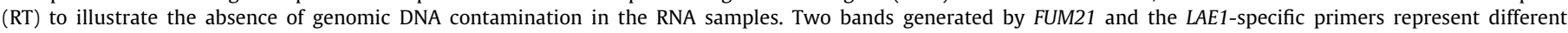
alternative splice forms. Total RNA from a 6 day culture of wild-type strain M-3125 was used as a positive control. 
(A) Fumonisin LC-MS analysis $\left(\mathrm{m} / \mathrm{z}=722\right.$, full scale $\left.=2 \times 10^{8}\right)$

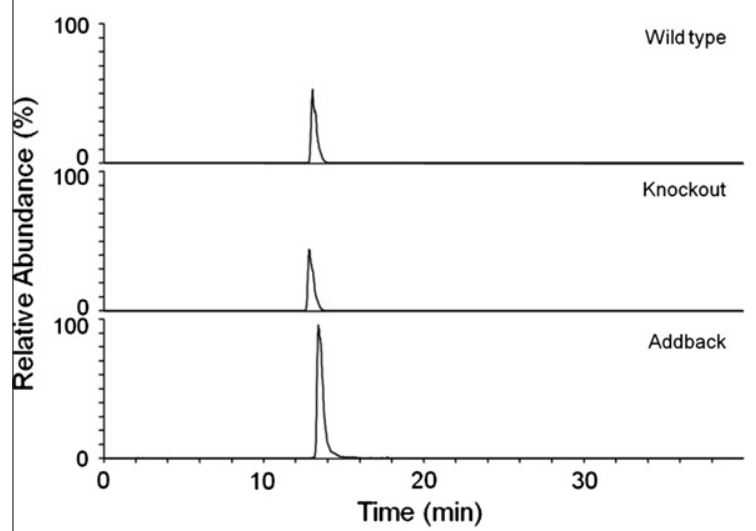

(B) Bikaverin analysis LC-MS/MS analysis (parent ion $\mathrm{m} / \mathrm{z}=383$, fragment ion $\mathrm{m} / \mathrm{z}=354$, full scale $=1.16 \times 10^{7}$ )

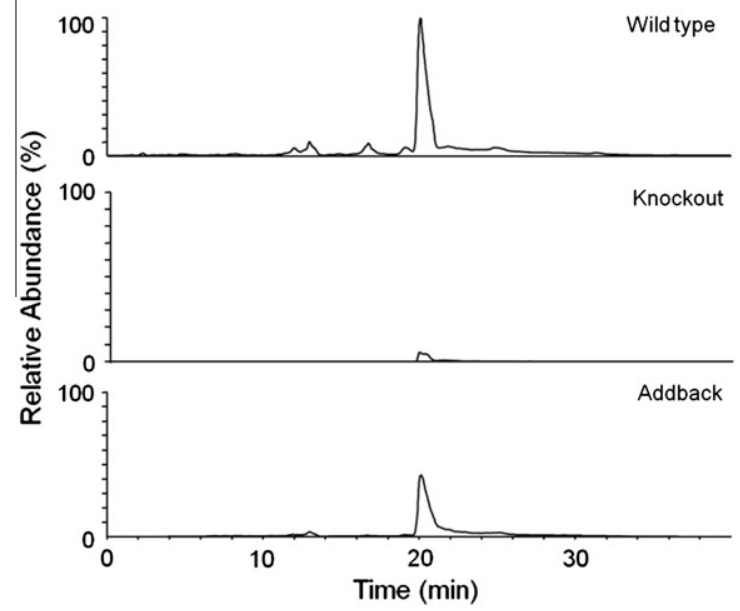

(C) Fusarin C analysis LC-MS/MS analysis (fragment ion $\mathrm{m} / \mathrm{z}=381$, parent ion $\mathrm{m} / \mathrm{z}=432$, full scale $=8.24 \times 10^{3}$ )

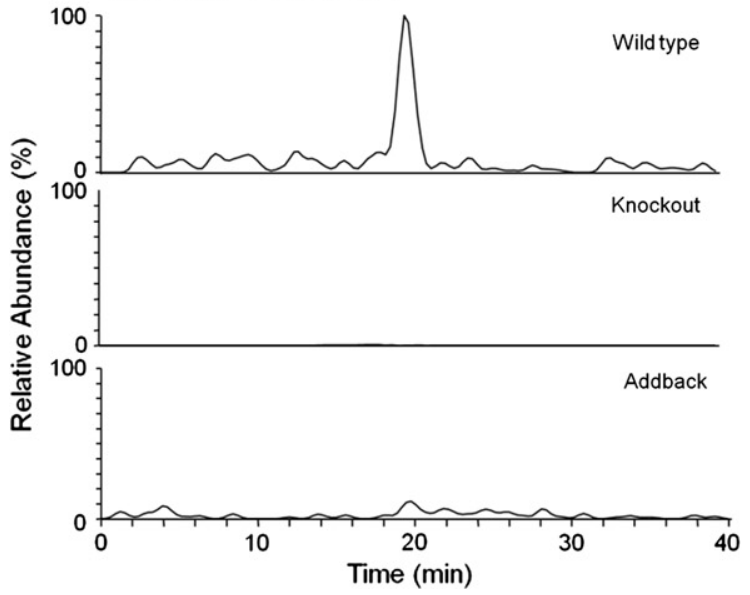

Fig. 7. Representative chromatograms for analysis after $F$. verticillioides growth in liquid GYAM (A and C) or on cracked corn (B). Wild type isolate is M-3125, knockout out is T22.1, addback is GMTcp-263. (A) LC-MS fumonisin analysis from 6 day liquid GYAM cultures, $m / z=722$, full scale $=2 \times 10^{8}$. (B) LC-MS/MS bikaverin analysis from 11 day crack corn cultures, parent ion $m / z=383$, fragment ion $m /$ $z=354$, full scale $=1.16 \times 10^{7}$. (C) LC-MS/MS fusarin C analysis from 6 day liquid GYAM cultures, fragment ion $m / z=381$, parent ion $m / z=432$, full scale $=$ $8.24 \times 10^{3}$. gene cluster containing a PKS (FVEG_00079). In the case of the only other PKS containing gene cluster whose product has been determined, the PGL gene cluster required for perithecial pigment production (Gaffor et al., 2005; Graziani et al., 2004; Proctor et al., 2007), the expression of the genes in this cluster were uncoordinated in the lae 1 mutant, that is, two of the genes including the PKS were expressed 2-fold less, two genes had no change in expression while two others were expressed more than 4 -fold higher than wild-type (data not shown). The PGL gene cluster likely contains six genes based on coordinate expression in the wild-type strain M-3125 when cultured on liquid GYAM media (Brown et al., 2012b).

Here, we have shown that deletion of the $F$. verticillioides LAE1 homolog results in altered expression of multiple SM gene clusters, both positive and negative, and a reduction in production of the SMs bikaverin and fusarin C. Despite decreased expression of FUM cluster genes, fumonisin production in the lae 1 mutant strain was not significantly reduced. The observation that the LAE1 complemented strain produced $50 \%$ more fumonisins than wild-type indicates that LAE1 affects fumonisin production in addition to FUM gene transcription. The differential effect on fumonisin production by lae 1 mutants as compared to FvVe1 mutants in F. verticillioides is similar to the differential effect on bikaverin production by respective $F$. fujikuroi mutants (Wiemann et al., 2010). In contrast, we found that the effect on bikaverin production in the lae1 mutants differed from $F$. fujikuroi LAE1 mutants. In the $F$. verticillioides lae 1 mutant, BIK gene expression and bikaverin production was greatly reduced while in the F. fujikuroi LAE1 mutant, BIK gene expression and bikaverin production were only slightly reduced (Wiemann et al., 2010). A comparison of these studies in $F$. fujikuroi and $F$. verticillioides demonstrate that the velvet complex, although conserved among fungi, can have different effects on production of the same SM in closely related species. Previously, the $V e A$ homolog FvVe1 in F. verticillioides was shown to positively affect production of two SMs, fumonisins and fusarins (Myung et al., 2009). In F. fujikuroi, all three components of the velvet complex have been described to affect SM synthesis as well as sexual and asexual development and pathogenicity on rice plants. FfVel1 and FfVel2 positively affect the production of gibberellic acids, fumonisins and fusarin $C$ and negatively affect the production of bikaverin. FfLae1 has a positive effect on gibberellins and bikaverin production (Wiemann et al., 2010). A Fusarium homolog of VelB was identified and described by Wiemann et al. (2010) in F. fujikuroi as FfVel2. Nucleotide sequence similarity search by BLAST of the F. verticillioides genome database with FfVel2 revealed the homolog as FVEG_01498. Expression of this gene in the F. verticillioides lae1 mutant was not different than in the wild-type strain.

Microarray analysis of gene expression in the lae 1 mutant and wild-type $F$. verticillioides revealed differential expression of two novel gene clusters. Although the SMs that are synthesized by the enzymes encoded by the genes in these clusters are not known, the identification of the genes in a putative cluster can be a critical first step in identification of such metabolites. Work with the laeA deletion strain in Aspergillus species led to the identification of multiple new SM gene clusters as well as the corresponding metabolites (Bok et al., 2006b; Bouhired et al., 2007; Perrin et al., 2007). Further exploration of the expression patterns in the lae1 mutant under different growth conditions in $F$. verticillioides could yield insight into previously undiscovered gene clusters and potential metabolites. Similarly, overexpression of LAE1 in F. verticillioides might also aid in the identification of novel SM gene clusters. We have recently described the phylogenomics of the $F$. verticillioides PKSs and discussed domain analysis as it can be applied to predicting the types of natural products expected from these gene clusters (Brown et al., 2012a).

The regulation of expression of SM biosynthetic gene clusters is proving to be complex. Visentin et al. (2012) have recently 
described the epigenetic regulation of a FUM1 promoter:green fluorescent protein gene fusion in $F$. verticillioides. They conclude that histone modification of the promoter region of FUM genes is involved in transcriptional regulation. The role of A. nidulans LaeA in regulation histone acetylation has been described (ReyesDominguez et al., 2010). Furthermore, many environmental and nutritional factors affect gene expression via broad-domain transcription factors. In $F$. verticillioides, nitrogen regulatory elements, $\mathrm{pH}$ regulatory elements as well as sugar transporters affect FUM gene cluster expression (Bluhm and Woloshuk, 2006; Bluhm et al., 2008; Flaherty et al., 2003; Kim and Woloshuk, 2008). In Aspergillus, genes that regulate conidia and ascospore production also affect secondary metabolite pathways (Bayram and Braus, 2011 and references therein). The discovery of the velvet complex has revealed yet another level of regulation of gene expression: i.e. regulation via changes in heterochromatin structure.

It is unlikely that all fungal SMs have been identified. The number of putative SM gene clusters in both Aspergillus and Fusarium exceed the number of SMs characterized in each of these fungi (Amaike and Keller, 2011; Ma et al., 2010). One highly successful strategy to determine the function of some of these cryptic SM gene clusters has been to force their expression by deleting or overexpressing different components of the velvet complex (Bok et al., 2006b; Bouhired et al., 2007). The effects of either strategy are unpredictable but consistent; some gene clusters require LAE1 for expression, while others are expressed in its absence (Bok et al., 2006b and the present work). Understanding the effects of the velvet complex on SM biosynthesis by Fusarium will allow a more complete understanding of how Lae1 and associated proteins regulate production of known metabolites and aid in the identification of new metabolites produced by the numerous other gene clusters present in Fusarium genomes.

\section{Acknowledgments}

We thank Crystal E. Probyn and Debbie Shane for technical assistance and Robert H. Proctor for critical evaluation of the manuscript. Mention of trade names or commercial products in this article is solely for the purpose of providing specific information and does not imply recommendation or endorsement by the US Department of Agriculture. USDA is an equal opportunity provider and employer.

\section{Appendix A. Supplementary material}

Supplementary data associated with this article can be found, in the online version, at http://dx.doi.org/10.1016/j.fgb.2012.06.003.

\section{References}

Amaike, S., Keller, N.P., 2011. Aspergillus flavus. Annu. Rev. Phytopathol. 49, 107 133.

Bayram, O., Braus, G.H., 2011. Coordination of secondary metabolism and development in fungi: the velvet family of regulatory proteins. FEMS Microbiol. Rev.. http://dx.doi.org/10.1111/j.1574-6976.2011.00285.x.

Bayram, Ö., Krappmann, S., Ni, M., Jin, W.B., Helmstaedt, K., Valerius, O., et al., 2008 VelB/VeA/LaeA complex coordinates light signal with fungal development and secondary metabolism. Science 320 (5882), 1504-1506.

Bluhm, B.H., Woloshuk, C.P., 2006. Fck1, a C-type cyclin-dependent kinase, interact with Fcc1 to regulate development and secondary metabolism in Fusarium verticillioides. Fungal Genet. Biol. 43 (3), 146-154.

Bluhm, B.H., Kim, H., Butchko, R.A., Woloshuk, C.P., 2008. Involvement of ZFR1 of Fusarium verticillioides in kernel colonization and the regulation of FST1, a putative sugar transporter gene required for fumonisin biosynthesis on maize kernels. Mol. Plant Pathol. 9 (2), 203-211.

Bok, J.W., Keller, N.P., 2004. LaeA, a regulator of secondary metabolism in Aspergillus spp. Eukaryot. Cell 3 (2), 527-535.

Bok, J.W., Noordermeer, D., Kale, S.P., Keller, N.P., 2006a. Secondary metabolic gene cluster silencing in Aspergillus nidulans. Mol. Microbiol. 61 (6), 1636-1645.
Bok, J.W., Hoffmeister, D., Maggio-Hall, L.A., Murillo, R., Glasner, J.D., Keller, N.P., 2006b. Genomic mining for Aspergillus natural products. Chem. Biol. 13 (1), 3137.

Bouhired, S., Weber, M., Kempf-Sontag, A., Keller, N.P., Hoffmeister, D., 2007. Accurate prediction of the Aspergillus nidulans terrequinone gene cluster boundaries using the transcriptional regulator LaeA. Fungal Genet. Biol. 44 (11), 1134-1145.

Brown, D.W., Cheung, F., Proctor, R.H., Butchko, R.A.E., Zheng, L., Lee, Y., et al., 2005 Comparative analysis of 87,000 expressed sequence tags from the fumonisinproducing fungus Fusarium verticillioides. Fungal Genet. Biol. 42, 848-861.

Brown, D.W., Butchko, R.A.E., Busman, M., Proctor, R.H., 2007. The Fusarium verticillioides FUM gene cluster encodes a Zn(II)2Cys6 protein that affects FUM gene expression and fumonisin production. Eukaryot. Cell 6, 1210-1218.

Brown, D.W., Butchko, R.A.E., Proctor, R.H., 2008. Genomic analysis of Fusarium verticillioides. Food Addit. Contam. Part A 25, 1158-1165.

Brown, D.W., Butchko, R.A.E., Baker, S.E., Proctor, R.H., 2012a. Phylogenomic and functional domain analysis of polyketide synthases in Fusarium. Fungal Biol. $116,318-331$

Brown, D.W. et al., 2012b. Identification of gene clusters associated with fusaric acid, fusarin, and perithecial pigment production in Fusarium verticillioides. Fungal Genet. Biol. http://dx.doi.org/10.1016/j.fgb.2012.05.010.

Busman, M., Butchko R.A.E., Proctor, R.H., submitted for publication. Utilization of high performance liquid chromatography coupled to tandem mass spectrometry for characterization of bikaverin production by species of Fusarium. J. Chromatogr. B: Analyt. Technol. Biomed. Life Sci.

Butchko, R.A.E., Adams, T.H., Keller, N.P., 1999. Aspergillus nidulans mutants defective in stc gene cluster regulation. Genetics 153, 715-720.

Butchko, R.A.E., Plattner, R.D., Proctor, R.H., 2003. FUM13 encodes a short-chain dehydrogenase/reductase required for $\mathrm{C}-3$ carbonyl reduction during fumonisin biosynthesis in Gibberella moniliformis. J. Agric. Food Chem. 51, 3000-3006.

Butchko, R.A.E., Plattner, R.D., Proctor, R.H., 2006. Deletion analysis of FUM genes involved in tricarballylic ester formation during fumonisin biosynthesis. J. Agric. Food Chem. 54, 9398-9404.

Calvo, A.M., 2008. The VeA regulatory system and its role in morphological and chemical development in fungi. Fungal Genet. Biol. 45, 1053-1061.

Colvin, B.M., Cooley, A.J., Beaver, R.W., 1993. Pulmonary edema and hydrothorax in swine produced by fumonisin B1, a toxic metabolite of Fusarium moniliforme. J. Vet. Diagn. Invest. 5, 232-241.

Cooks, R.G., Rockwood, A.L., 1991. The 'Thomson'. A suggested unit for mass spectroscopists. Rapid Commun. Mass Spectrom. 5 (2), 93.

Desjardins, A.E., Plattner, R.D., Proctor, R.H., 1996. Linkage among genes responsible for fumonisin biosynthesis in Gibberella fujikuroi mating population A. Appl. Environ. Microbiol. 62, 2571-2576.

Doko, M.B., Rapior, S., Visconti, A., Schjoth, J.E., 1995. Incidence and levels of fumonisin contamination in maize genotypes grown in Europe and Africa. J. Agric. Food Chem. 43, 429-434.

Edgar, R., Domrachev, M., Lash, A.E., 2002. Gene Expression Omnibus: NCBI gene expression and hybridization array data repository. Nucleic Acids Res. 30 (1), 207-210.

Flaherty, J.E., Woloshuk, C.P., 2004. Regulation of fumonisin biosynthesis in Fusarium verticillioides by a zinc binuclear cluster-type gene, ZFR1. Appl. Environ. Microbiol. 70, 2653-2659.

Flaherty, J.E., Pirttilä, A.M., Bluhm, B.H., Woloshuk, C.P., 2003. PAC1, a pH-regulatory gene from Fusarium verticillioides. Appl. Environ. Microbiol. 69 (9), 52225227.

Franceschi, S., Bidoli, E., Baron, A.E., La Vecchia, C., 1990. Maize and risk of cancers of the oral cavity, pharynx, and esophagus in northeastern Italy. J. Natl. Cancer Inst. 82, 1407-1411.

Gaffor, I., Brown, D.W., Plattner, R., Proctor, R.H., Qi, W., Trail, F., 2005. Functional analysis of polyketide synthase genes in the filamentous fungus Gibberella zeae (anamorph Fusarium graminearum). Eukaryot. Cell 4 (11), 1926-1933.

Graziani, S., Vasnier, C., Daboussi, M.-J., 2004. Novel polyketide synthase from Nectria haematococca. Appl. Environ. Microbiol. 70 (5), 2984-2988.

Harrison, L.R., Colvin, B.M., Green, J.T., Newmann, L., Cole, J.R., 1990. Pulmonary edema and hydrothorax in swine produced by fumonisin B1, a toxic metabolic of Fusarium moniliforme. J. Vet. Diagn. Invest. 2, 217-221.

Hoff, B., Kamerewerd, J., Sigl, C., Mitterbauer, R., Zadra, I., Kürnsteiner, H., et al., 2010. Two components of a velvet-like complex control hyphal morphogenesis, conidiophore development, and penicillin biosynthesis in Penicillium chrysogenum. Eukaryot. Cell 9 (8), 1236-1250.

Irizarry, R.A., Hobbs, B., Collins, F., Beazer-Barclay, Y.D., Antonellis, K.J., Scherf, U., et al., 2003. Exploration, normalization, and summaries of high density oligonucleotide array probe level data. Biostatistics 4, 249-264.

Kim, H., Woloshuk, C.P., 2008. Role of AREA, a regulator of nitrogen metabolism, during colonization of maize kernels and fumonisin biosynthesis in Fusarium verticillioides. Fungal Genet. Biol. 45 (6), 947-953.

Kleigrewe, K., Söhnel, A.-C., Humpf, H.-U., 2011. A new high-performance liquid chromatography-tandem mass spectrometry method based on dispersive solid phase extraction for the determination of the mycotoxin fusarin $C$ in corn ears and processed corn samples. J. Agric. Food Chem. 59 (19), 10470-10476.

Kroken, S., Glass, N.L., Taylor, J.W., Yoder, O.C., Turgeon, B.G., 2003. Phylogenetic analysis of type I polyketide synthase genes in pathogenic and saprobic ascomycetes. Proc. Natl. Acad. Sci. USA 100, 15670-15675.

Leslie, J.F., Plattner, R.D., Desjardins, A.E., Klittich, C.J.R., 1992. Fumonisin B 1 production by strains from different mating populations of Gibberella fujikuroi (Fusarium section Liseola). Phytopathology 82, 341-345. 
Li, S., Myung, K., Guse, D., Donkin, B., Proctor, R.H., Grayburn, W.S., et al., 2006. FvVE1 regulates filamentous growth, the ratio of microconidia to macroconidia and cell wall formation in Fusarium verticillioides. Mol. Microbiol. 62, 14181432.

Linnemannstöns, P., Schulte, J., del Mar Prado, M., Proctor, R.H., Avalos, J., Tudzynski, B., 2002. The polyketide synthase gene pks4 from Gibberella fujikuroi encodes a key enzyme in the biosynthesis of the red pigment bikaverin. Fungal Genet. Biol. 37, 134-148.

Ma, L.J. et al., 2010. Comparative genomics reveals mobile pathogenicity chromosomes in Fusarium. Nature 464 (7287), 367-373.

Maragos, C.M., Busman, M., Plattner, R.D., 2008. Development of monoclonal antibodies for the fusarin mycotoxins. Food Addit. Contam. 25 (1), 105114.

Marasas, W.F.O., 1996. Fumonisins: history, world-wide occurrence and impact. In: Jackson, L.S., DeVaries, W., Bullerman, L.B. (Eds.), Fumonisins in Food. Plenum Publishing Co., New York, NY.

Marek, E.T., Schardl, C.L., Smith, D.A., 1989. Molecular transformation of Fusarium solani with an antibiotic resistance marker having no fungal DNA homology. Curr. Genet. 15, 421-428.

Myung, K., Li, S., Butchko, R.A.E., Busman, M., Proctor, R.H., Abbas, H.K., Calvo, A.M., 2009. FvVE1 regulates biosynthesis of the mycotoxins fumonisins and fusarins in Fusarium verticillioides. J. Agric. Food Chem. 57 (11), 5089-5094.

Oswiler, G.D., Ross, P.F., Wilson, T.M., Nelson, P.E., Witte, S.T., Carson, T.L., et al., 1992. Characterization of an epizootic of pulmonary edema in swine associated with fumonisin in corn screenings. J. Vet. Diagn. Invest. 4, 53-59.

Perrin, R.M., Fedorova, N.D., Jin, W.B., Cramer Jr., R.A., Wortman, J.R., Kim, H.S., et al. 2007. Transcriptional regulation of chemical diversity in Aspergillus fumigatus by LaeA. PLoS Pathog. 3 (4), 508-517.

Plattner, R.D., Weisleder, D., Poling, S.M., 1996. Analytical determination of fumonisins and other metabolites produced by Fusarium moniliforme and related species on corn. In: Jackson, L.S., DeVaries, W., Bullerman, L.B. (Eds.), Fumonisins in Food. Plenum Press, New York.

Proctor, R.H., Desjardins, A.E., Plattner, R.D., 1999a. Biosynthetic and genetic relationships of B-series fumonisins produced by Gibberella fujikuroi mating population A. Nat. Toxins 7, 251-258.

Proctor, R.H., Desjardins, A.E., Plattner, R.D., Hohn, T.M., 1999b. A polyketide synthase gene required for biosynthesis of fumonisin mycotoxins in Gibberella fujikuroi mating population A. Fungal Genet. Biol. 27, 100-112.

Proctor, R.H., Brown, D.W., Plattner, R.D., Desjardins, A.E., 2003. Co-expression of fifteen contiguous genes delineates a fumonisin biosynthetic gene cluster in Gibberella moniliformis. Fungal Genet. Biol. 38, 237-249.

Proctor, R.H., Butchko, R.A.E., Brown, D.W., Moretti, A., 2007. Functional characterization, sequence comparisons and distribution of a polyketide synthase gene required for perithecial pigmentation in some Fusarium species. Food Addit. Contam. Part A 24, 1076-1087.
Reyes-Dominguez, Y., Bok, J.W., Berger, H., Shwab, E.K., Basheer, A., Gallmetzer, A., et al., 2010. Heterochromatic marks are associated with the repression of secondary metabolism clusters in Aspergillus nidulans. Mol. Microbiol. 76 (6), $1376-1386$.

Rheeder, J.P. Marasas, W.F.O., Thiel, P.G. Sydenham, E.W. Shepard, G.S., Van Schalkwyk, D.J., 1992. Fusarium moniliforme and fumonisins in corn in relation to human esophageal cancer in Transkei. Phytopathology 82, 353-357.

Sambrook, J., Fritsch, E.F., Maniatis, T., 1989. Molecular Cloning: A Laboratory Manual, second ed. Cold Spring Harbor Laboratory Press, Cold Spring Harbor NY

Seo, J.-A., Proctor, R.H., Plattner, R.D., 2001. Characterization of four clustered and coregulated genes associated with fumonisin biosynthesis in Fusarium verticillioides. Fungal Genet. Biol. 34, 155-165.

Song, Z., Cox, R.J., Lazarus, C.M., Simpson, T.J., 2004. Fusarin C biosynthesis in Fusarium moniliforme and Fusarium venenatum. ChemBioChem 5, 1196-1203.

Suarez, L., Hendricks, K.A., Cooper, S.P., Sweeny, A.M., Hardy, R.J., Larsen, R.D., 2000 Neural tube defects among Mexican Americans living on the US-Mexico border: effects of folic acid and dietary folate. Am. J. Epidemiol. 152, 1017-1023.

Sydenham, E.W., Thiel, P.G., Marasas, W.F.O., Shephard, G.S., Schalkwyk, D.J. Koch, K.R., 1990. Natural occurrence of some Fusarium mycotoxins in corn from low and high esophageal cancer prevalence areas of the Transkei, Southern Africa. J Agric. Food Chem. 38, 1900-1903.

Vesonder, R., Haliburton, J., Golinski, P., 1989. Toxicity of field samples and Fusarium moniliforme from feed associated with equine-leucoencephalomalacia. Arch. Environ. Contam. Toxicol. 18 (3), 439-442.

Visentin, I. Montis, V., Doll, K, Alabouvetter, C., Tamietti, G., Karlovsky, P. Cardinale, F., 2012. Transcription of genes in the biosynthetic pathway for fumonisin mycotoxins is epigenetically and differentially regulated in the fungal maize pathogen Fusarium verticillioides. Eukaryot. Cell 11, 252-259.

Wiemann, P., Willmann, A., Straeten, M., Kleigrewe, K., Beyer, M., Humpf, H.U., et al. 2009. Biosynthesis of the red pigment bikaverin in Fusarium fujikuroi: genes, their function and regulation. Mol. Microbiol. 72 (4), 931-946.

Wiemann, P., Brown, D.W., Kleigrewe, K., Bok, J.W., Keller, N.P., Humpf, H.-U., et al. 2010. FfVel1 and FfLae1, components of a velvet-like complex in Fusarium fujikuroi, affect differentiation, secondary metabolism and virulence. Mol Microbiol. 77 (4), 972-994.

Yoshizawa, T., Yamashita, A., Luo, Y., 1994. Fumonisin occurrence in corn from highand low-risk areas for human esophageal cancer in China. Appl. Environ. Microbiol. 60, 1626-1629.

Yu, J.-H., Keller, N.P., 2005. Regulation of secondary metabolism in filamentous fungi. Annu. Rev. Phytopathol. 43, 437-458.

Zaleta-Rivera, K., Xu, C., Yu, F., Butchko, R.A.E., Proctor, R.H., Hidalgo-Lara, M.E., Raza, A., et al., 2006. A bidomain nonribosomal peptide synthetase encoded by FUM14 catalyzes the formation of tricarballylic esters in the biosynthesis of fumonisins. Biochemistry 45, 2561-2569. 